EPJ manuscript No.

(will be inserted by the editor)

\title{
Aspects of $\phi$-meson production in proton-proton collisions
}

\author{
A. Sibirtsev ${ }^{1}$, J. Haidenbauer ${ }^{2}$ and U.-G. Meißner ${ }^{1,2}$ \\ 1 Helmholtz-Institut für Strahlen- und Kernphysik (Theorie), Universität Bonn, Nußallee 14-16, D-53115 Bonn, Germany \\ ${ }^{2}$ Institut für Kernphysik (Theorie), Forschungszentrum Jülich GmbH, D-52425 Jülich, Germany
}

Received: date / Revised version: date

\begin{abstract}
We analyze near-threshold cross section data for the reaction $p p \rightarrow p p \phi$ published by the DISTO collaboration and recent, still preliminary results presented by the ANKE Collaboration. We formulate a procedure to evaluate the OZI ratio at low energies by taking into account corrections from the kinematics and the final-state interaction. Combining the new data with the few measurements available at higher energies we give a limit for the OZI rule violation and estimate the possible contribution from a five-quark baryonic resonance coupled to the $\phi p$ system.
\end{abstract}

PACS. 13.60.Le Meson production - 14.20.Gk Baryon resonances with $S=0-12.10 . \mathrm{Qk}$ Unification of couplings 12.38.Qk Experimental tests

\section{Introduction}

$\phi$-meson production in hadronic reactions motivates both experimental and theoretical activities for several reasons. A well known argument is the possible violation of the Okubo-ZweigIizuka rule [ $\left.\begin{array}{lll}1 & 2 & 3\end{array}\right]$ and the role of the strangeness content of the nucleon. Moreover, $\phi p$ production allows to search for cryptoexotic baryons with hidden strangeness.

The OZI rule states that the production of open as well as hidden strangeness from any initial state that does not contain a $s \bar{s}$ component is strongly suppressed relative to the production of non-strange states. Therefore, studies of the OZI rule are generally based on considering measurements of corresponding $\phi$-meson and $\omega$-meson production reactions. The cross section ratio, $R_{\phi / \omega}$, is then compared with the limit proposed by Lipkin [4]. The latter follows from SU(3) symmetry and involves the experimentally known deviation $\Delta \theta_{V}=3.7^{\circ}$ from the ideal mixing angle between singlet and octet vector mesons, i.e.

$$
\begin{array}{r}
R_{\phi / \omega}=\frac{g_{\phi \rho \pi}^{2}}{g_{\omega \rho \pi}^{2}}=\frac{g_{\phi N N}^{2}}{g_{\omega N N}^{2}}=\frac{\sigma(\pi N \rightarrow \phi X)}{\sigma(\pi N \rightarrow \omega X)}=\frac{\sigma(N N \rightarrow \phi X)}{\sigma(N N \rightarrow \omega X)} \\
=\tan ^{2}\left(\Delta \theta_{V}\right)=4.2 \cdot 10^{-3}
\end{array}
$$

where the $g$ 's denote corresponding coupling constants and $X$ stands for any inclusive final state that does not contain strange quarks. Actually Eq. (1) is already violated on the level of the $\phi \rho \pi$ and $\omega \rho \pi$ coupling constants that can be extracted from $\phi \rightarrow \rho \pi, \omega \rightarrow 3 \pi, \omega \rightarrow \pi \gamma$ and $\rho \rightarrow \pi \gamma$ decays [5 6]. These decays [7] result in an average ratio $R_{\phi / \omega}=(12.5 \pm 3.4) \cdot 10^{-3}$. Note that is also established [8] that the $\phi \rightarrow \rho \pi$ decay alone violates OZI rule.

A systematic analysis [9] of experiments on $\phi$ and $\omega$ production in $\pi N$ and $N N$ reactions led to a ratio of $R_{\phi / \omega}=$
$(13.4 \pm 3.2) \cdot 10^{-3}$. Apparently, all phenomenological models $\left.\begin{array}{llllll}10 & 11 & 12 & 13 & 14\end{array}\right]$ that directly incorporate the $\phi \rho \pi$ and $\omega \rho \pi$ vertices would be able to reproduce the large ratio given above. The same is also true for $N N \rightarrow N N \phi / \omega$ calculations [15 16] which utilize $\pi N \rightarrow \phi N$ and $\pi N \rightarrow \omega N$ transition amplitudes fitted to the data. Therefore, values around $R_{\phi / \omega} \simeq 13 \cdot 10^{-3}[9]$ are not necessarily related to the strangeness content of the nucleon. Only ratios significantly larger than this value might be considered as a possible indication of a $s \bar{s}$ component of the initial state.

A very recent analysis [17] of $\phi$ and $\omega$ photoproduction from the proton shows that the ratio $R_{\phi / \omega}=0.8 \pm 0.2$ at photon energies above $30 \mathrm{GeV}$. This was interpreted in terms of quark-anti-quark fluctuations of the photon. Considering the $u \bar{u}, d \bar{d}, s \bar{s}, c \bar{c}, b \bar{b}$ and $t \bar{t}$ photon structure one might expect that at high energies, i.e. in the perturbative QCD regime, the ratios of different vector mesons approach unity, up to corrections due to the hadronic wave functions. The analysis [18 19] of $\omega$ and $J / \Psi$ photoproduction results in the ratio $R_{[J / \Psi] / \omega}=0.04 \pm 0.01$ at photon energy around $3 \cdot 10^{3} \mathrm{GeV}$, i.e. at the maximal energy where $\omega$-meson photoproduction data are available. We concluded that the large $\phi / \omega$ and $[J / \Psi] / \omega$ ratios observed in photoproduction at high energies are related to the nature of the photon itself but are irrelevant for the strangeness content of the nucleon. It was also noted that the $\phi$ photoproduction at low photon energies and for large four-momentum transfer squared $t$ could not be understood in terms of perturbative QCD and probably the large ratio $R_{\phi / \omega} \simeq 0.1$ at $|t|>2 \mathrm{GeV}^{2}$ can be only explained if one assumes a large ratio of the $\phi N N$ and $\omega N N$ couplings. Modern dispersion analyses [20 21] of the nucleon electromagnetic form factors show that the squared ratio of these coupling constant is about $\simeq 0.23$ (provided one assumes that the whole strength in the spectral function at $t \simeq 1 \mathrm{GeV}^{2}$ is entirely given by the $\phi$-meson, therefore such values should be considered as upper limits). 
There are still some phenomena [22] which could be a signal of a $s \bar{s}$ component in the nucleon. For example, protonantiproton annihilation at rest results in $R_{\phi / \omega}=0.294 \pm 0.097$ for the $\phi \gamma$ and $\omega \gamma$ final states while a ratio $R_{\phi / \omega}=0.106 \pm 0.012$ was found for the $\phi \pi$ and $\omega \pi$ channels. At the same time the available data for the annihilation in flight yield a ratio $R_{\phi / \omega}$ $=(14.55 \pm 1.92) \cdot 10^{-3}$, which is compatible with the ratios extracted from $\pi N$ and $N N$ reactions and from vector meson decays. Again a substantial violation of the OZI rule was detected at low energies.

These observations in the $\gamma p$ and $\bar{p} p$ reactions provide a strong motivation to further search for substantial OZI rule violations at low energies. In 1998, the DISTO Collaboration reported a low energy $p p \rightarrow p p V$ measurement indicating that the ratio $R_{\phi / \omega}$ is enhanced by about an order of magnitude relative to the OZI limit given in Eq. (1). The experiment was done at a proton beam energy of $T_{l a b} \simeq 2.85 \mathrm{GeV}$, i.e. at an excess energy $\epsilon \simeq 82 \mathrm{MeV}$ above the $p p \rightarrow p p \phi$ reaction threshold. At such low energies corrections due to the differences in the $p p \rightarrow p p \phi$ and $p p \rightarrow p p \omega$ phase space and the $p p$ final state interaction (FSI) should be implemented for a meaningful analysis with regard to an OZI rule violation. Indeed Eq. (1) holds only for high energies and when the cross sections are practically energy independent.

Since the corrections mentioned above were not fully implemented in the analysis of Ref. [24] the results given by the DISTO collaboration can be only considered as qualitative estimations. Here we describe a procedure for the data evaluation that allows for an analysis of any 3-body final state. Following that procedure we extract the ratio $R_{\phi / \omega}$ from the DISTO results and from preliminary $p p \rightarrow p p \phi$ cross section data reported recently by the ANKE Collaboration [25]. Note that the evaluation of the ratio $R_{\phi / \omega}$ requires data for $p p \rightarrow p p \omega$ as well. High-statistics $\omega$ data were published recently by the TOF Collaboration [26 27]. Those data allow us to extract the $p p \rightarrow p p \omega$ reaction amplitude, which can be considered an added bonus of our present study.

The manuscript is organized as follows. In Sec.[2] we present and discuss our method to extract the OZI ratio from data on vector meson production in proton-proton collisions. It is applied to data from Saclay and COSY and the results are presented in Sec. 3 Sec. 4 is devoted to the possible coupling of exotic states to the $\phi$-proton final state. We end with a summary and outlook in Sec.5

\section{Method for extracting the OZI ratio from proton-proton collisions}

Using non-relativistic 3-body phase space and applying corrections due to the FSI between the protons, the average $p p \rightarrow p p \omega$ reaction amplitude $|\mathcal{M}|^{2}$ can be extracted from the reaction cross section $\sigma$ by means of [28]

$$
\begin{aligned}
\sigma(\epsilon)= & \frac{\sqrt{m_{N}^{2} m_{\omega}}}{2^{7} \pi^{2}\left(2 m_{N}+m_{\omega}\right)^{3 / 2}} \frac{\epsilon^{2}}{\sqrt{s^{2}-4 s m_{N}^{2}}} \\
& \times\left[1+\frac{4 \beta^{2}-4 \alpha^{2}}{-\alpha+\sqrt{\alpha^{2}+m_{N} \epsilon}}\right]|\mathcal{M}|^{2},
\end{aligned}
$$
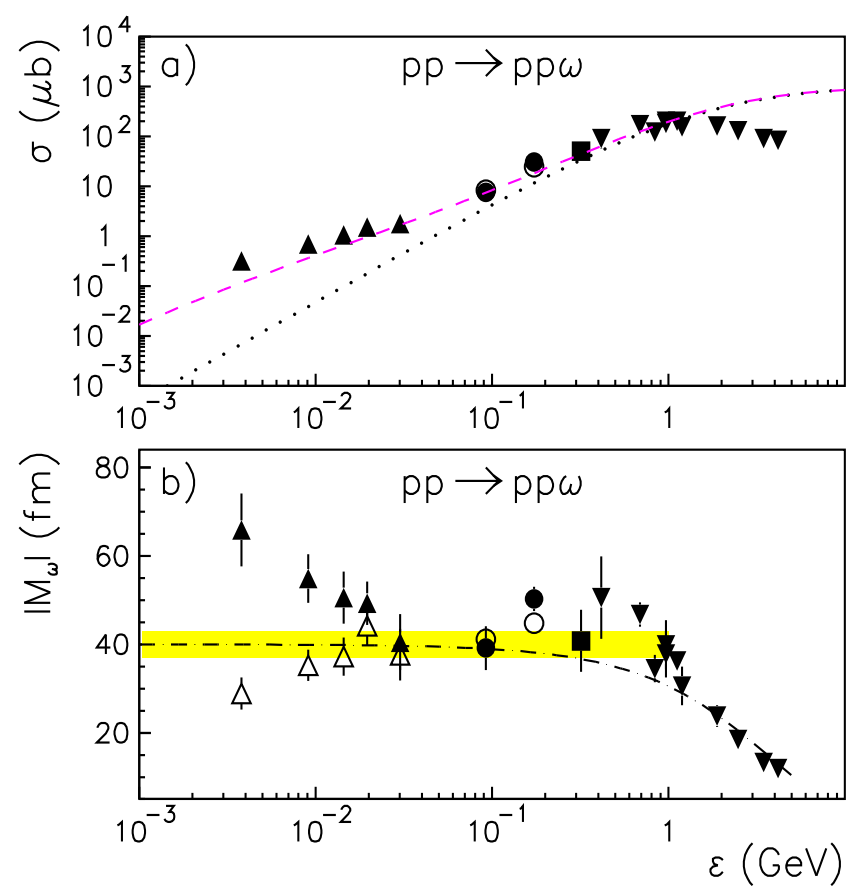

Fig. 1. (a) The $p p \rightarrow p p \omega$ cross section and (b) the average reaction amplitude $\left|\mathcal{M}_{\omega}\right|^{2}$ as a function of the excess energy. The cross section data are from Refs. [23] (solid inverse triangles), [24] (solid squares), [29] (solid triangles), [26] (solid circles), and [27] (open circles), respectively. The corresponding values in (b) are extracted from the cross section data via Eq. [2]. The open triangles correspond to the data of Ref. 29] but using Eq. 5. The dashed line corresponds to Eq. 2] with $|\mathcal{M}|=40 \mathrm{fm}$, while the dotted line indicates the results without FSI corrections. The hatched area indicates the average amplitude used in the present paper for the OZI analysis. The dash-dotted line is a phenomenological fit to the data of the form $\mathcal{M}=40 \mathrm{fm} \exp (-0.27 \epsilon)$.

where $\epsilon=\sqrt{s}-2 m_{N}-m_{\omega}, \sqrt{s}$ is the invariant collision energy given in terms of the proton beam kinetic energy $T_{l a b}$ by $s=$ $2 m_{N}\left(2 m_{N}+T_{l a b}\right)$ and $m_{N}, m_{\omega}$ are the nucleon and $\omega$-meson masses, respectively. The FSI effects are taken into account by means of the Jost function method with

$$
|J(q)|^{-1}=\frac{q+i \beta}{q-i \alpha}=\left[\frac{r \beta^{2}}{2}+\frac{r q^{2}}{2}\right]\left[-\frac{1}{a}+\frac{r q^{2}}{2}-i q\right]^{-1},
$$

which at small $q$ goes over into the Watson-Migdal parameterization and at large $q$ approaches unity. In Eq. (3) $\alpha$ and $\beta$ are the parameters that specify the FSI between the protons; they are related to the scattering parameters by

$$
a=\frac{\alpha+\beta}{\alpha \beta}, \quad r=\frac{2}{\alpha+\beta}
$$

with $\alpha<0$ and $\beta>0$. In the present study we use the values $\alpha=$ $-20.5 \mathrm{MeV} / \mathrm{c}$ and $\beta=166.7 \mathrm{MeV} / \mathrm{c}$. Integrating the square of the Jost function (3) over the nonrelativistic 3-body phase space yields the factor in front of $|\mathcal{M}|^{2}$ in Eq. (2).

The presently available data for the $p p \rightarrow p p \omega$ reaction cross

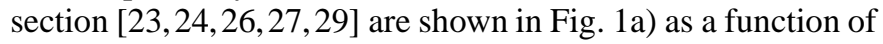
the excess energy. Applying Eq. (2) we can extract the average 
reaction amplitude $|\mathcal{M}|$. Corresponding results are displayed in Fig. 1b). To evaluate the $\phi / \omega$ ratio for the DISTO and ANKE data we need the $p p \rightarrow p p \omega$ reaction amplitude for excess energies in the range $10<\epsilon<100 \mathrm{MeV}$. The only data available in this energy range are those of the TOF [26 27] and SPESIII [29] collaborations. The reaction amplitude extracted from the SPES-III data via Eq. (2) exhibits a strong energy dependence near threshold which might be due to (neglecting) the finite width of the $\omega$ meson. Indeed one should account for the $\omega$ width, $\Gamma=8.49 \mathrm{MeV}$, when analyzing the data at low excess energies, i.e. at $\epsilon \simeq \Gamma$. In that case the relation between the $p p \rightarrow p p \omega$ cross section and reaction amplitude is given as

$$
\begin{gathered}
\sigma(\epsilon)=\frac{1}{2^{8} \pi^{3} s \sqrt{s^{2}-4 s m_{N}^{2}}} \int_{2 m_{\pi}}^{\sqrt{s}-2 m_{N}} \frac{d x}{2 \pi} \frac{\Gamma|\mathcal{M}|^{2}}{\left(x-m_{\omega}\right)^{2}+\Gamma^{2} / 4} \\
\int_{4 m_{N}^{2}}^{(\sqrt{s}-x)^{2}} d y \frac{\sqrt{y^{2}-4 y m_{N}^{2}} \sqrt{(s-y-x)^{2}-4 y x^{2}}}{y} \frac{y^{2}-4 m_{N}^{2}+4 \beta^{2}}{y^{2}-4 m_{N}^{2}+4 \alpha^{2}}
\end{gathered}
$$

Replacing the $\omega$-meson spectral distribution by the $\delta$-function in Eq. (5) one recovers Eq. (2) in the non-relativistic limit. Indeed the relativistic corrections play only a minor role at $\epsilon<10 \mathrm{GeV}$, which can be checked by numerical integration of Eq. (5). The $p p \rightarrow p p \omega$ reaction amplitude extracted from SPES-III data utilizing Eq. (5) is shown by the open triangles in Fig. 1b). Although again the amplitude deviates from a constant at small $\epsilon$ we interpolate it as $|\mathcal{M}|=(40 \pm 3)$ fm for our further OZI analysis. Finally the dashed line in Fig. 1h) shows the result of Eq. (2) with $|\mathcal{M}|=40 \mathrm{fm}$, while the dotted line indicates the results without FSI corrections, i.e. with $\alpha=\beta$ in Eq. (2).

We would like to make a short comment concerning the FSI corrections, or more precisely concerning the normalization of the lines in Fig. 11). Since we choose $|\mathcal{M}|=40 \mathrm{fm}$ we reasonably describe the data for $\epsilon \leq 1 \mathrm{GeV}$. But the use of a constant matrix element in Eq. (2) apparently leads to an overestimation of the cross section at high energies. However, one should keep in mind that any phenomenological model for that reaction will contain form factors that depend on the squared fourmomentum transfer from the initial to the final proton. This $t$-dependence becomes substantial [28] at $\epsilon>1 \mathrm{GeV}$ and effectively reduces the value of the integral over the phase space. This in turn allows to describe data at high energies within such models [15 16].

\section{Results}

The formalism for extracting the reaction amplitude $|\mathcal{M}|^{2}$ for $\phi$-meson production is the same as described in the preceeding section, except that now the $\phi$ mass, $m_{\phi}$, appears in Eq. (2) and the excess energy is defined by $\epsilon=\sqrt{s}-2 m_{N}-m_{\phi}$. Fig. 2 shows the $p p \rightarrow p p \phi$ cross section and the ratio of the $\phi$ and $\omega$ production amplitude, $\left|\mathcal{M}_{\phi}\right|^{2} /\left|\mathcal{M}_{\omega}\right|^{2}$, as a function of the excess energy. In general, there are no measurements available for $\omega$ as well as $\phi$ production at exactly the same $\epsilon$ and, in addition, the $\omega$-meson data shown in Fig. 1 indicate some fluctuations,
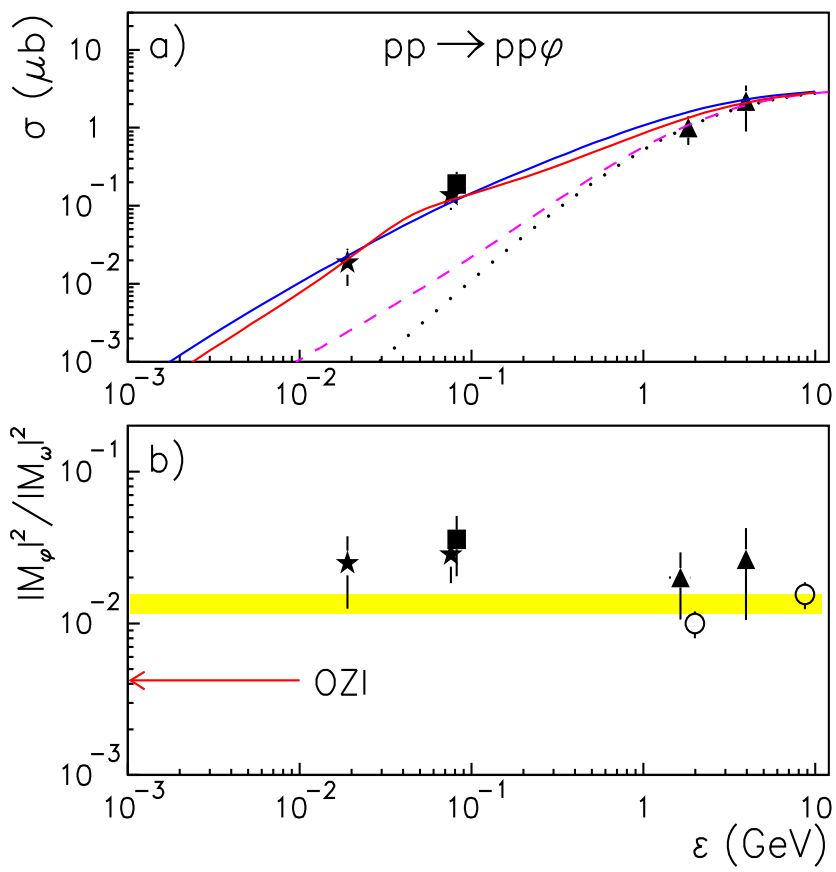

Fig. 2. (a) The $p p \rightarrow p p \phi$ cross section and (b) the ratio of the amplitudes $\left|\mathcal{M}_{\phi}\right|^{2} /\left|\mathcal{M}_{\omega}\right|^{2}$ as a function of the corresponding excess energy. The square is the result from DISTO [24], the stars are the preliminary ANKE data [25], while the triangles are from Refs. [23 30]. The open circles in (b) are from ratio measurements in the forward hemisphere [31 32]. The dashed line shows the result of Eq. [2] with $|\mathcal{M}|=2.3 \mathrm{fm}$, while the dotted line indicates the results without FSI corrections. The solid lines are the results obtained with the inclusion of a baryonic resonance coupled to $\phi p$ system, discussed in Sect. 4. The hatched area indicates the ratio extracted previously [9] from a combined $\pi N$ and $N N$ OZI analysis. The arrow indicates the OZI limit given in Eq. 1 .

which might be related to systematics. Thus, for calculating the ratio for the DISTO and ANKE data we use the average amplitude $|\mathcal{M}|=(40 \pm 3) \mathrm{fm}$ for $\omega$ meson production. The triangles in Fig. (2b) show the ratios obtained from the data on the total cross sections of Refs. [23 30]. The open circles are ratios published in Refs. [31 32] which correspond to cross section measurements in the forward hemisphere. Note that at such high energies differences in the phase space are practically negligible.

The hatched band in Fig. 20 $\mathrm{b}$ ) indicates the ratio $R_{\phi / \omega}=$ $(13.4 \pm 3.2) \cdot 10^{-3}$ extracted from a combined analysis of $\pi N$ and $N N$ data in Ref. [9], which is close to the value $R_{\phi / \omega}=$ $(12.5 \pm 3.4) \cdot 10^{-3}$ obtained from the vector meson decays. The DISTO measurement results in the ratio $R_{\phi / \omega}=(36 \pm 16) \cdot 10^{-3}$. The still preliminary ANKE data [25] are consistent with the DISTO result. Thus, the central value of the ratio is indeed a factor of 4 larger than what was found in the other analyses. Unfortunately, however, the systematic uncertainties of the data on the $\phi / \omega$ ratio from $p p$ collisions are rather large and, therefore, impede the claim for a clear and more substantial OZI rule violation as compared to the limits found, for instance, in vector meson decays. 
In any case, it is clear that the near-threshold cross section data for the reaction $p p \rightarrow p p \phi$ are enhanced as compared to the measurements at $\epsilon>1 \mathrm{GeV}$. This is demonstrated by the dashed line in Fig. 2a). It corresponds to a calculation using Eq. (2) and utilizing a constant reaction amplitude, $|\mathcal{M}|$ $=2.3 \mathrm{fm}$, which is normalized to the high energy data. In order to demonstrate that more clearly let us now use Eq. (2) to extract the reaction amplitude $|\mathcal{M}|$ from the measured cross sections. Corresponding results are shown in Fig. 3 To facilitate a comparision with the energy dependence of the amplitude for the reaction $p p \rightarrow p p \omega$ we include here a fit to that amplitude (dash-dotted line), taken over from Fig. 11 Given the few data available and the large error bars there is some freedom in how the curve for $\omega$ production is superimposed on the results for the $\phi$ production amplitude. Thus, at present one cannot rule out that the $\epsilon$-dependence of the $p p \rightarrow p p \phi$ amplitude is basically the same as the one obtained for the reaction $p p \rightarrow p p \omega$. On the other hand, the data do suggest that, in the range $0.1<\epsilon<1 \mathrm{GeV}$, the $p p \rightarrow p p \phi$ amplitude exhibits a significantly stronger variation with energy than what is seen for the $\omega$ production. This characteristic energy dependence could be a sign for an additional reaction mechanism in the $\phi$ production reaction, and specifically it might be caused by the excitation of a resonance in the $\phi p$ system. We will elaborate on this point in the next section.

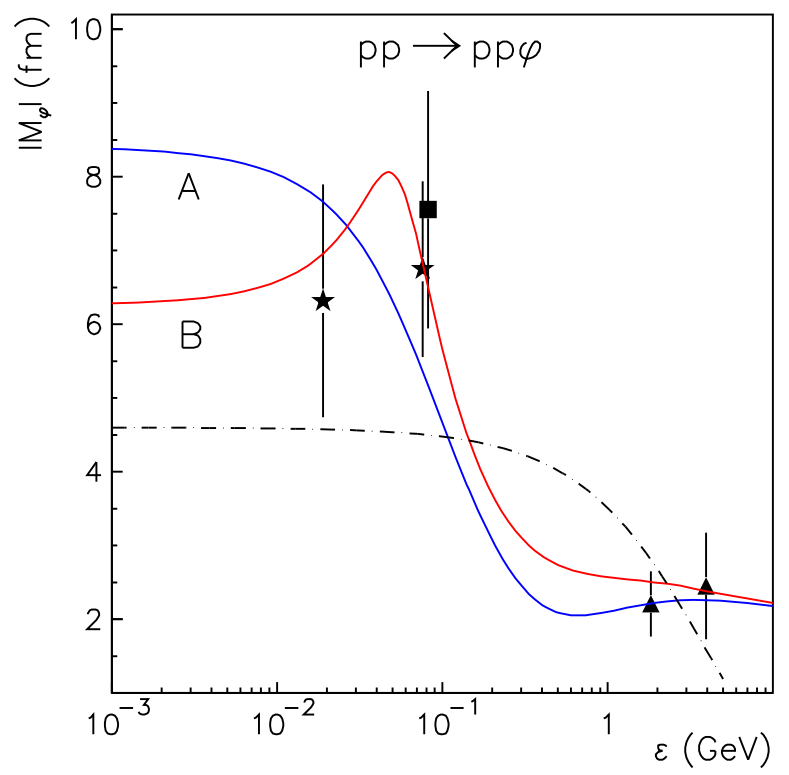

Fig. 3. The $p p \rightarrow p p \phi$ reaction amplitude, $\left|\mathcal{M}_{\phi}\right|$, as a function of the excess energy $\epsilon$. The square is the result extracted from the DISTO data [24], the stars are those for the preliminary ANKE data [25], while the triangles are from Refs. [23 30]. The lines show results of a calculation that includes a baryonic resonance in the $\phi p$ system with the parameters sets A and B given by Eq. 7). The dash-dotted line indicates the energy dependence of the $p p \rightarrow p p \omega$ amplitude and is taken over from Fig. 1]
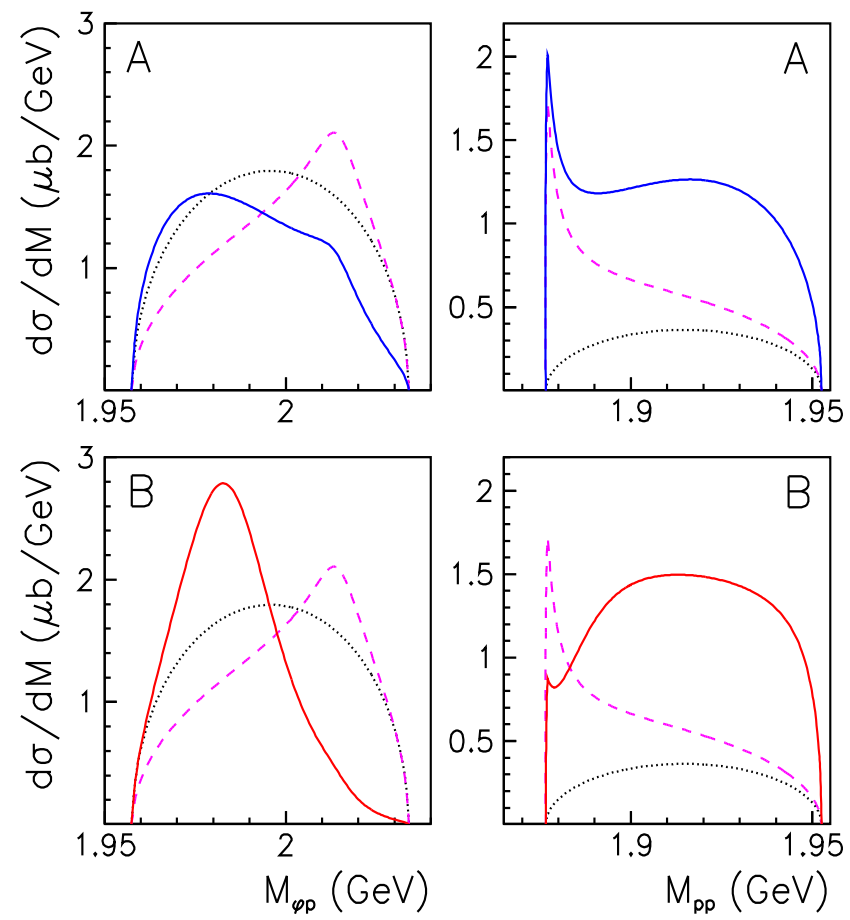

Fig. 4. The $\phi p$ and $p p$ invariant mass spectra for the reaction $p p \rightarrow$ $p p \phi$ at $\epsilon=76 \mathrm{MeV}$. The dotted lines show the phase space distributions, the dashed lines are calculations with inclusion of the $p p$ FSI and the solid lines are results obtained with including the $p p$ FSI and a $B_{\phi}$ pentaquark resonance with parameter sets $\mathrm{A}$ and B from Eq. (7).

\section{Speculations on exotic baryons}

A few years ago Landsberg proposed [33 34] that $\phi p$ production, which is OZI suppressed for non-resonant reactions, is well suited for the search of cryptoexotic baryons with hidden strangeness, $B_{\phi}=u d d s \bar{s}$. It is expected that these pentaquark baryons have a narrow width and decay preferentially into the $\phi N, K \bar{K} N$ or $Y K$ channels, where $Y$ stands for ground-state or excited hyperons. Note that these decays are OZI allowed. Experimental limits for the $B_{\phi}$ candidates were reported in Refs. [31,35 36 37, 38 39]. There are two independent observations of a narrow peak in the $\Sigma(1385)^{0} K^{+}$spectra, the first one with a mass $M=2050 \pm 6 \mathrm{MeV}$ and width $\Gamma \leq 50 \pm 19$ $\mathrm{MeV}$ [37] and the second one with $M=1956_{-6}^{+8} \overline{\mathrm{MeV}}$ and $\Gamma=27 \pm 15 \mathrm{MeV}$ [35]. The high-statistics study of Ref. [39] of the $\Sigma^{0} K^{+}$mass spectrum indicates two exotic states with $M=1807 \pm 7 \mathrm{MeV}, \Gamma=62 \pm 19 \mathrm{MeV}$ and $M=1986 \pm 6$ $\mathrm{MeV}, \Gamma=91 \pm 20 \mathrm{MeV}$.

If cryptoexotic $B_{\phi}$ baryons indeed exist one expects them to contribute to the $p p \rightarrow p p \phi$ reaction. That was the basic idea of the experiment of Ref. [31] where an upper limit for $B_{\phi} \rightarrow \phi p$ was provided but the low statistics did not allow to draw definite conclusions from the $\phi p$ mass spectrum. Note that these measurements were done at $\epsilon \simeq 2 \mathrm{GeV}$, where the non-resonant contribution to the process $p p \rightarrow p p \phi$ might dominate, as can be estimated from Fig. 3 In that sense a study of a possible $B_{\phi}$ contribution at low excess energies has advantages and we expect that COSY is well suited for such an investigation [40]. 
To estimate the possible effect from a $B_{\phi}$ state, we introduce a baryonic resonance in the $\phi p$ subsystem and parameterize the reaction amplitude by

$$
\mathcal{M}=\mathcal{M}_{0}+\frac{c_{0} e^{i \phi} M \Gamma}{M^{2}-s_{\phi p}-i M \Gamma},
$$

where $M$ and $\Gamma$ are the resonance mass and width, respectively, while $s_{\phi p}$ is the squared invariant mass of the $\phi p$ subsystem. Here we take $\mathcal{M}_{0}=3 \mathrm{fm}$, in line with the data at higher energies, cf. Fig. 3 and adjust the real constant $c_{0}$ so that the $p p \rightarrow p p \phi$ data are reproduced over the whole considered energy range. The possible phase $\phi$ between the two amplitudes was chosen to be $\phi=0$ for convenience (note also that the existing data do not allow to pin down this phase). The curves in Fig. 3 correspond to calculations with two different parameter sets:

$$
\begin{aligned}
& \mathrm{A}: M=1956 \mathrm{MeV}, \Gamma=120 \mathrm{MeV}, c_{0}=8 \mathrm{fm} \\
& \mathrm{B}: \quad M=2000 \mathrm{MeV}, \Gamma=40 \mathrm{MeV}, c_{0}=8 \mathrm{fm}
\end{aligned}
$$

Obviously it is not possible to determine the $B_{\phi}$ parameters unambiguously from the ANKE and the DISTO data on the reaction cross section. Therefore, we present results from two different sets which describe the data almost equally well. A reasonable choice of $B_{\phi}$ allows to reproduce the data on $p p \rightarrow$ $p p \phi$ cross section, as illustrated by the two solid lines in Fig. 2a). It is clear that, in principle, the large $p p \rightarrow p p \phi$ cross section at low energies could be explained by a $B_{\phi}$ excitation.

However, this is rather speculative and further detailed studies are necessary before conclusions can be drawn. First it is important to have the final results from the ANKE Collaboration [40] with high statistical accuracy and also at low excess energies, where the different $B_{\phi}$ resonances yield different energy dependences of the $p p \rightarrow p p \phi$ reaction cross section. It would be also important to get data for the $p p \rightarrow p p \phi$ reaction at higher energies to verify the strong energy dependence of the reaction amplitude in the region $0.1<\epsilon<2 \mathrm{GeV}$, and specifically to map out the energy dependence in that region in detail. This could e.g. be done at the JINR Nuclotron [41].

Independently of that, a direct investigation of a possible $B_{\phi}$ contribution can be done through the analysis of the $\phi p$ and $p p$ invariant mass spectra. Fig. 4 shows results for these mass spectra for the $p p \rightarrow p p \phi$ reaction at $\epsilon=76 \mathrm{MeV}$, i.e. for the current experimental setup of ANKE. The dotted lines represent the phase space distribution with $|\mathcal{M}|=$ const., while the dashed lines are calculations where the $p p$ FSI is taken into account via Eq. (3). The solid lines in Fig. 47are predictions that include the $p p$ FSI and a $B_{\phi}$ resonance with parameter sets A and $\mathrm{B}$ given in Eq. (7). It is clear that the identification of a $B_{\phi}$ contribution requires high statistical accuracy and a high mass resolution. These are prerequisites for any experiment with the aim to resolve the issue of the possible existence of exotic pentaquark baryons with hidden strangeness.

\section{Summary and outlook}

In this paper, we have studied aspects of vector meson production in proton-proton collisions. We have proposed a strategy that allows to extract the OZI ratio $R_{\phi / \omega}$ correcting for the kinematical differences in the processes $p p \rightarrow p p \omega$ and $p p \rightarrow p p \phi$, respectively, and for the final-state interactions. The resulting values collected in Fig. 2 are somewhat larger but still compatible with the extraction of $R_{\phi / \omega}$ from $\pi N$ and $N N$ reactions. We have also pointed out a strong energy dependence of the $p p \rightarrow p p \phi$ reaction amplitude for excess energies $0.1<\epsilon<1 \mathrm{GeV}$. This might be indicative of a resonance coupling strongly to the $\phi p$ system. We have presented results assuming the presence of a cryptoexotic baryon with hidden strangeness. However, to establish such a state, a precise measurement of invariant mass distributions, see Fig. 44 is mandatory. Such measurements could be performed with the ANKE detector at COSY.

\section{Acknowledgements}

We would like to thank K.-Th. Brinkmann, H.-W. Hammer, C. Hanhart, M. Hartmann, Y. Maeda, J. Ritman and E. Roderburg for useful discussions. This work was partially supported by Deutsche Forschungsgemeinschaft through funds provided to the SFB/TR 16 "Subnuclear Structure of Matter". This research is part of the EU Integrated Infrastructure Initiative Hadron Physics Project under contract number RII3-CT-2004506078. A.S. acknowledges support by the COSY FFE grant No. 41760632 (COSY-085).

\section{References}

1. S. Okubo, Phys. Lett. 5, 165 (1963).

2. G. Zweig, CERN report TH-412 (1964).

3. J. Iizuka, Prog. Theor. Phys. Suppl. 38, 21 (1966).

4. H.J. Lipkin, Phys. Rev. 60B, 371 (1976).

5. M. Gell-Mann and F. Zachariasen, Phys. Rev. 124, 953 (1961).

6. U.-G. Meißner, Phys. Rep. 161, 213 (1988)

7. S. Eidelman et al. (Particle Data Group), Phys. Lett. B 592, 1 (2004).

8. P. Jain, R. Johnson, U.-G. Meißner, N. W. Park and J. Schechter, Phys. Rev. D 37, 3252 (1988).

9. A. Sibirtsev and W. Cassing, Eur. Phys. J. A 7, 407 (2000), |nucl-th/9907059|.

10. K. Nakayama, J.W. Durso, J. Haidenbauer, C. Hanhart and J. Speth, Phys. Rev. C 60, 055209 (1999) |arXiv:nucl-th/9904040|.

11. K. Nakayama, J. Haidenbauer and J. Speth, Phys. Rev. C 63, 015201 (2001) arXiv:nucl-th/0008047.

12. A.I. Titov, B. Kämpfer and B.L. Reznik, Eur. Phys. J. A 7, 543 (2000) |arXiv:nucl-th/0001027|.

13. A.I. Titov, B. Kämpfer and B.L. Reznik, Phys. Rev. C 65, 065202 (2002) arXiv:nucl-th/0102032.

14. L.P. Kaptari and B. Kämpfer, Eur. Phys. J. A 23, 291 (2005) |arXiv:nucl-th/0406077|.

15. D. Griffiths, A.M. Saperstein and D.T. Schnitzler, Nucl. Phys. B 34, 397 (1971).

16. A.A. Sibirtsev, Nucl. Phys. A 604, 455 (1996).

17. A. Sibirtsev, U.-G. Meißner and A.W. Thomas, Phys. Rev. D 71, 094011 (2005) |arXiv:hep-ph/0503276|.

18. A. Sibirtsev, K. Tsushima and S. Krewald, Phys. Rev. C 67, 055201 (2003) arXiv:nucl-th/0301015.

19. A. Sibirtsev, S. Krewald and A.W. Thomas, J. Phys.G 30, 1427 (2004) |arXiv:nucl-th/0301082|. 
20. H.W. Hammer and U.-G. Meißner, Eur. Phys. J. A 20, 469 (2004) |arXiv:hep-ph/0312081|.

21. P. Mergell, U.-G. Meißner and D. Drechsel, Nucl. Phys. A 596, 367 (1996) |arXiv:hep-ph/9506375|.

22. V.P. Nomokonov and M.G. Sapozhnikov, Phys. Part. Nucl. 34, 94 (2003) |arXiv:hep-ph/0204259|.

23. Landolt-Börnstein, New Series, Springer, I/12 (1998).

24. F. Balestra et al., Phys. Rev. Lett. 81, 4572 (1998); F. Balestra et al., Phys. Rev. C 63, 024004 (2001).

25. M. Hartmann et al., Nucl. Phys. A 755, 459 (2005).

26. S. Abd El-Samad et al., Phys. Lett. B 522, 16 (2001).

27. M. Schulte-Wissermann, Diploma Thesis, University of Dresden (2004).

28. A. Sibirtsev, J. Haidenbauer, H.-W. Hammer and S. Krewald, in preparation.

29. F. Hibou et al., Phys. Rev. Lett. 83, 492 (1999).
30. V. Blobel et al., Nucl. Phys. B 69, 237 (1974).

31. M.W. Arenton et al., Phys. Rev. D 25, 2241 (1982)

32. S.V. Golovkin et al., Z. Phys. A 359, 435 (1997).

33. L.G. Landsberg, Phys. Usp. 37, 1043 (1994).

34. L.G. Landsberg, Phys. Rep. 320, 223 (1999).

35. A.N. Aleev et al., Z. Phys. C 25, 205 (1984).

36. V.A. Dorofeev et al., Phys. Atom. Nucl. 57, 227 (1994).

37. V.A. Dorofeev et al., Phys. Atom. Nucl. 57, 238 (1994).

38. M.Ya. Balatz et al., Z. Phys C 61, 223 (1994).

39. Yu.M. Antipov et al., Phys. Atom. Nucl. 65, 2070 (2002).

40. M. Hartmann et al., COSY Proposal 104.2 (2004).

41. R.A. Salmin et al., in Proceedings of the International Conference on High-Energy Interactions: Theory and Experiment (Hadron Structure '02), edited by J. Urban et al. (Kosice Safarik Univ., Slovakia, 2003), p. 280. 